\title{
Proporção áurea na reabilitação de múltiplos diastemas com laminados vitrocerâmicos reforçados com dissilicato de lítio
}

Golden ratio in the rehabilitation of multiple diastemas with vitroceramic laminates reinforced with lithium disilicate Proporción áurea en la rehabilitación de múltiples diastemas con laminados vitrocerámicos reforzados con disilicato de litio

Paula Mayumi SIQUEIRA ${ }^{1}$

Fábio Eduardo de SIQUEIRA ${ }^{\mathbf{1}}$ Edson SUGUIAMA ${ }^{1}$

Gabriel Marcondes CASTANHEIRA ${ }^{2}$

Fernanda Midori TSUZUKI ${ }^{2}$

Silvia Sbenghen Bicudo SÁBIO ${ }^{3}$

Carina Gisele Costa BISPO ${ }^{4}$

\begin{abstract}
${ }^{1}$ Mestre em Odontologia Integrada, Centro de Ciências da Saúde, Universidade Estadual de Maringá (UEM), 87080-000 Maringá - PR,Brasil ${ }^{2}$ Mestrando, Centro de Ciências da Saúde, Departamento de Odontologia, Universidade Estadual de Maringá (UEM), 87080-000 Maringá - PR,Brasil ${ }^{3}$ Doutora em Odontologia, Centro de Ciências da Saúde, Departamento de Odontologia, Universidade Estadual de Maringá (UEM), 87080-000 Maringá - PR,Brasil ${ }^{4}$ Doutora em Ciências Odontológicas, Centro de Ciências da Saúde, Departamento de Odontologia, Universidade Estadual de Maringá (UEM), 87080-000 Maringá - PR,Brasil
\end{abstract}

\begin{abstract}
Resumo
Introdução: O restabelecimento de um sorriso harmônico constitui um grande desafio para a odontologia restauradora. A sincronia entre diversas especialidades se faz necessária para diagnosticar e tratar alterações estéticas, buscando a excelência do resultado e a satisfação do paciente. Um método bastante citado na literatura e aplicado por muitos clínicos é baseado na teoria da regra de proporção áurea. Quando adotada para situações complexas a proporção áurea pode ser um ponto de referência para a reabilitação. Deste modo, o trabalho tem como objetivo demonstrar uma reabilitação estética de diastemas múltiplos após tratamento ortodôntico, por meio de laminados vitrocerâmicos reforçados com dissilicato de lítio, onde o conceito de proporção áurea é aplicado. Descrição do caso clínico: Primeiramente, o plano de tratamento consistiu na distribuição uniforme dos diastemas, sendo posicionados da maneira mais harmônica e favorável para a oclusão na reabilitação com laminados vitrocerâmicos. A segunda fase consistiu na cimentação dos laminados, a fim de obter um resultado estético agradável. Discussão: A associação de ortodontia prévia a instalação dos laminados, permite uma melhor harmonia estética e oclusal, garantindo uma longevidade do tratamento. Opções restauradoras como laminados vitrocerâmicos reforçados com dissilicato de lítio permitem reabilitações extensas minimamente invasivas. Para casos complexos a proporção áurea, quando alinhada com os conceitos de macro e microestética, pode levar ao sucesso estético.
\end{abstract}

Descritores: Estética Dentária; Facetas Dentárias; Diastema.

\section{Abstract}

Introduction: Set a harmonious smile consists in a great challenge for restorative dentistry. Synchronize between specialties be request to diagnose and treat aesthetic changes, seeking outcome excellence and patient satisfaction. Literature widely cited the golden proportion as a method applied by many clinicians. When adopted for complex situations the golden ratio can be a reference point for rehabilitation. We demonstrate a multiple diastema aesthetic rehabilitation after orthodontic treatment using lithium disilicate reinforced glass-ceramic laminates, applying the golden proportion. Description of the clinical case: First, treatment plan consisted in uniform distribution of diastemas, promoting the most harmonic and favorable position for the occlusion in glass-ceramic laminates rehabilitation. The second phase consisted of cementing the laminates in order to obtain a pleasant aesthetic result. Discussion: Prior orthodontic association installing laminates, allows for better aesthetic and occlusal harmony, ensuring longevity of the treatment. Restorative options such as glass-ceramic laminates with lithium disilicate allow minimally invasive extensive rehabilitation. In complex cases the golden proportion, aligned with the concepts of macro and microaesthetics, can lead to aesthetic success.

Descriptors: Esthetics, Dental; Dental Veneers; Diastema.

\section{Resumen}

Introducción: El restablecimiento de una sonrisa armónica constituye un gran desafío para la odontología restauradora. La sincronización entre diversas especialidades se hace necesaria para diagnosticar y tratar alteraciones estéticas, buscando la excelencia del resultado y la satisfacción del paciente. Un método bastante citado en la literatura y aplicado por muchos clínicos se basa en la teoría de la regla de proporción áurea. Cuando se adopta para situaciones complejas la proporción áurea puede ser un punto de referencia para la rehabilitación. De este modo, el trabajo tiene como objetivo demostrar una rehabilitación estética de díastemas múltiples tras tratamiento ortodóntico, por medio de laminados vitrocerámicos reforzados con disilicato de litio, donde el concepto de proporción áurea es aplicado. Descripción del caso clínico: Primero, el plan de tratamiento consistió en la distribución uniforme de los díastemas, siendo posicionados de la manera más armónica y favorable para la oclusión para rehabilitación con laminados vitrocerámicos. La segunda fase consistió en la cementación de los laminados, a fin de obtener un resultado estético agradable. Discusión: La asociación de ortodoncia previa la instalación de los laminados, permite una mejor armonía estética y oclusal, garantizando una longevidad del tratamiento. Las opciones restauradoras como laminados vitrocerámicos reforzados con disilicato de litio permiten rehabilitaciones extensas mínimamente invasivas. Para casos complejos la proporción áurea, cuando se alinea con los conceptos de macro y micro estética, puede llevar al éxito estético.

Descriptores: Estética Dental; Coronas con Frente Estético; Diastema.

INTRODUÇÃO

A percepção de um belo sorriso está conectada com os dentes anteriores, sendo importante a atenção à cor, forma e textura durante o processo restaurador $^{1}$. Os pacientes buscam melhorar as características do sorriso através de tratamento ortodôntico, restaurações estéticas, facetas e/ou clareamento $^{2}$. A sincronia entre diversas especialidades se faz necessária para diagnosticar e tratar alterações estéticas, buscando a excelência do resultado e a satisfação do paciente ${ }^{3}$. Dessa forma, laminados vitrocerâmicos minimamente invasivos são uma realidade extremamente atrativa e satisfatória para resolução de casos com comprometimento estético ${ }^{4}$.

A harmonia entre as dimensões dos dentes é um fator fundamental na aparência do sorriso, sendo dependente da relação altura/largura dental, disposições de cada elemento no arco, bem como a forma do arco e configuração do sorriso ${ }^{5}$. Portanto, o restabelecimento de um sorriso harmônico constitui 
um grande desafio para a odontologia restauradora. Um método bastante citado na literatura e aplicado por muitos clínicos é baseado na teoria da regra de proporção áurea ${ }^{6,7}$.

A proporção áurea, ou divina, é frequentemente aplicada na composição ideal dentária possibilitando resultados estéticos favoráveis ${ }^{8}$. Tal método não se limita a Dentística, podendo ser empregado na Ortodontia, Cirurgia Bucomaxilofacial e Prótese9. Quando adotada para situações complexas a proporção áurea pode ser um ponto de referência para a reabilitação. Ela é matematicamente definida como 1.0:0.62:0.38:0.24 ${ }^{10}$. Se aplicada de modo correto, o resultado da proporção áurea é naturalmente atrativo e agradável aos olhos ${ }^{3}$.

Deste modo, o trabalho tem como objetivo demonstrar uma reabilitação estética de diastemas múltiplos após tratamento ortodôntico, por meio de laminados vitrocerâmicos reforçados com dissilicato de lítio, onde o conceito de proporção áurea é aplicado.

\section{CASO CLÍNICO}

Paciente de 27 anos, sexo masculino, procurou a Clínica de Dentística da Associação Maringaense de Odontologia (AMO) para a finalização do tratamento ortodôntico através de reabilitação estética.

Durante o exame clínico, a análise estética revelou múltiplos diastemas nos arcos superior e inferior, incongruência entre os dentes, alterações de forma e tamanho. Os diastemas foram distribuídos de modo uniforme pelo ortodontista, sendo posicionados da maneira mais harmônica e favorável para a oclusão da reabilitação com laminados cerâmicos. Foi observado que o aparelho ortodôntico fixo estava em posição, mantendo o equilíbrio da mordida (Figura 1). Na reabilitação dos espaços interdentais foi utilizado vitro-cerâmica reforçada com dissilicato de lítio, IPS e.max CAD® (Ivoclar Vivadent Ltda., São Paulo, Brasil), para os dentes 11, 21, 12, 22, 13, $23,14,24,15,25,31,41,32,42,33,43,34,44,35$, e 45. A reabilitação do paciente se deu em dois momentos, sendo o arco superior reabilitado primeiro e o inferior em segundo lugar.

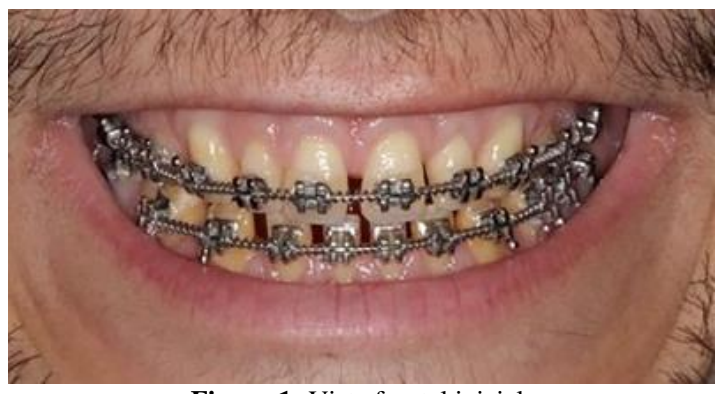

Figura 1: Vista frontal inicial.

Após a remoção do aparelho superior e moldagem, os dentes foram medidos para determinar as melhores proporções entre altura e largura utilizando o conceito de proporção áurea, por meio da grade de Levin. ${ }^{6}$ A grade foi posicionada de modo a sua linha média ficar entre os incisivos centrais, coincidindo com o plano sagital do paciente (Figura 2).

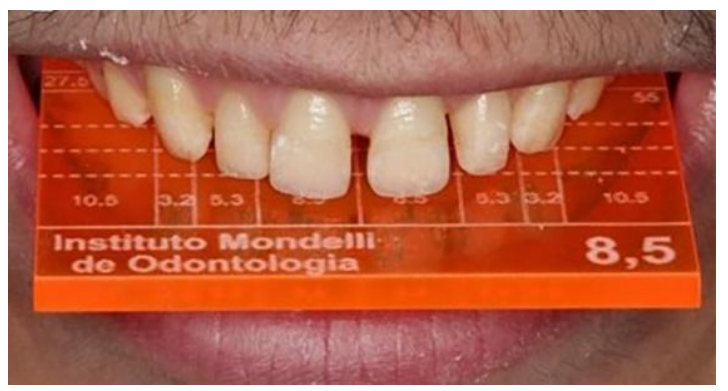

Figura 2: Planejamento com grade de Levin.

Com um compasso de ponta seca, foi medido o tamanho dos incisivos centrais direito e esquerdo separadamente, de modo que uma ponta foi posicionada no ponto de contato distal e outra no ponto mesial. O paciente foi encaminhado para a confecção de um aparelho removível a fim de manter a estabilidade dos espaços (Figura 3).

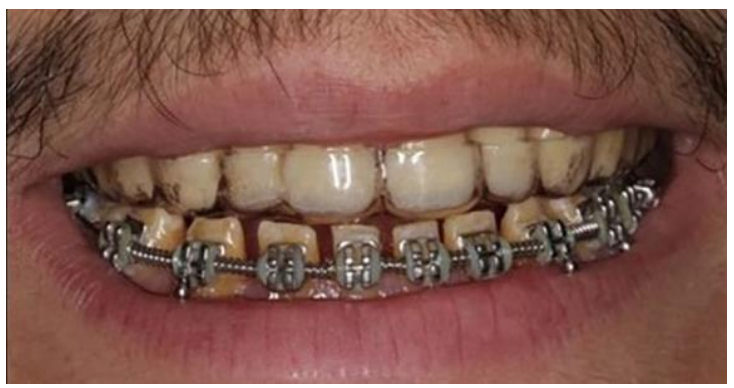

Figura 3: Mantenedor de espaço em posição.

De posse dos modelos de estudo e medidas estabelecidas, o laboratório produziu o enceramento diagnóstico sob o qual foi realizado o guia de silicone para o mock-up com resina bis acrílica, A1 (Protemp $^{\mathrm{TM}}$ 4, 3M ESPE, Brasil) do arco superior (Figuras 4 a 7 ).

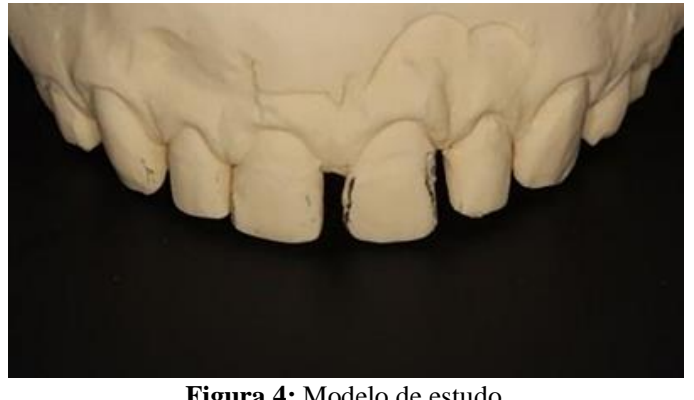

Figura 4: Modelo de estudo.

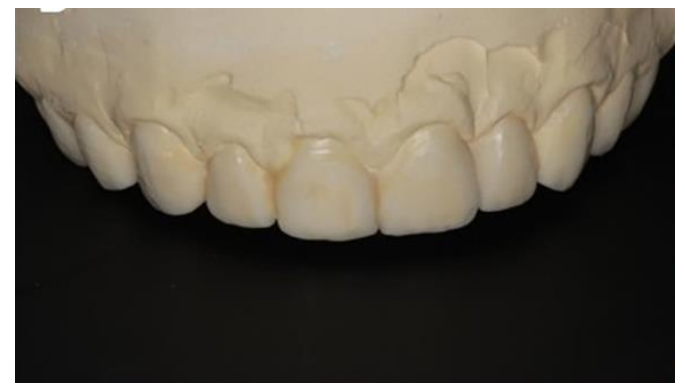

Figura 5: Enceramento diagnóstico. 


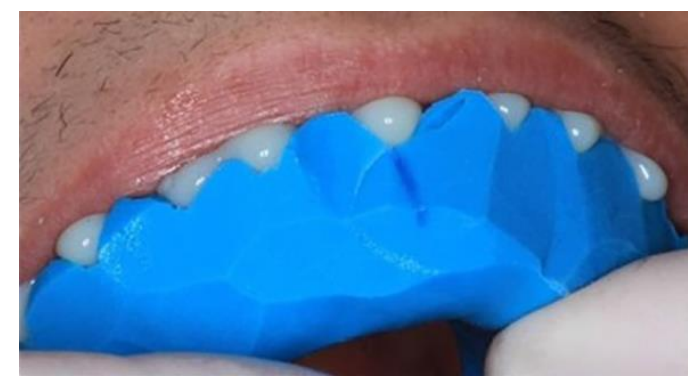

Figura 6: Guia de silicone para mock-up.

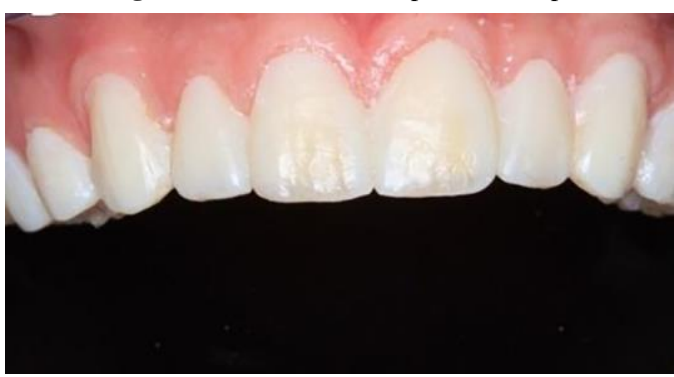

Figura 7: Mock-up confeccionado em resina bisacrílica

O preparo para os laminados dos elementos 15 ao 25 teve início com a remoção do mock-up, abrasão de pontos proeminentes, confecção de bisel discreto com pontas diamantadas (Figura 8). Ao final, nova moldagem foi efetuada com silicone de adição (Express $^{\mathrm{TM}}$ XT, 3M ESPE, Brasil), utilizando fio para afastamento gengival (Ultrapak®, Ultradent Products, Inc., São Paulo, Brasil) e tomada de cor dos dentes (Escala de Cores Vita Classical, Wilcos do Brasil Indústria e Comércio Ltda, Rio de Janeiro, Brasil), cor A2 (Figuras 9 a 12).

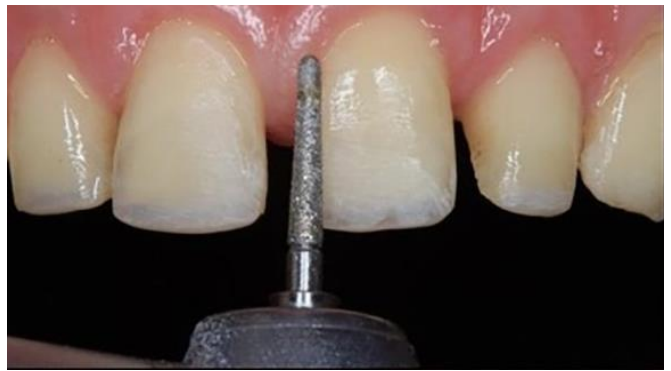

Figura 8: Confeccção de bisel.

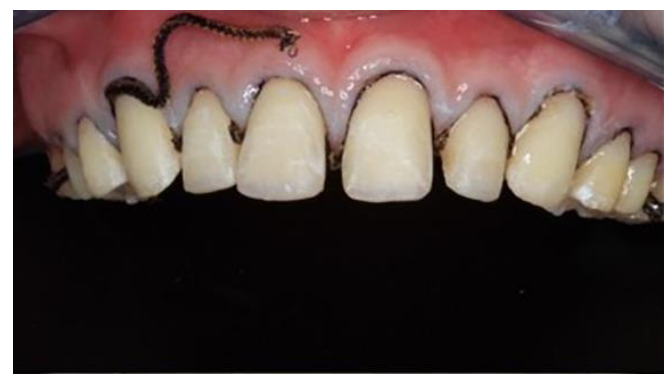

Figura 9: Afastamento gengival com fio retrator.

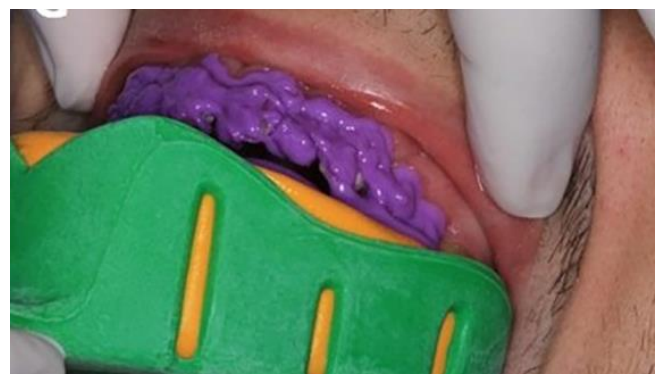

Figura 10: Moldagem com silicone de adição

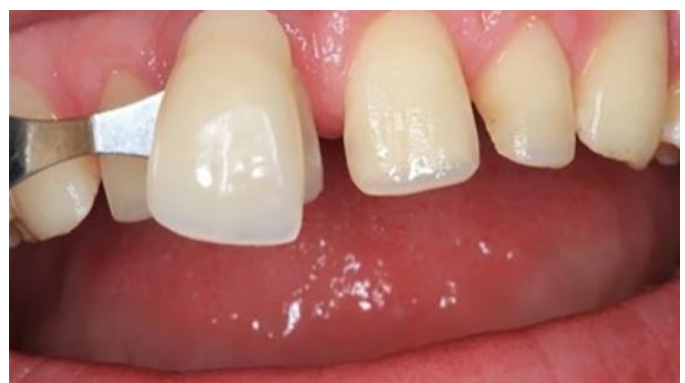

Figura 11: Seleção da cor.

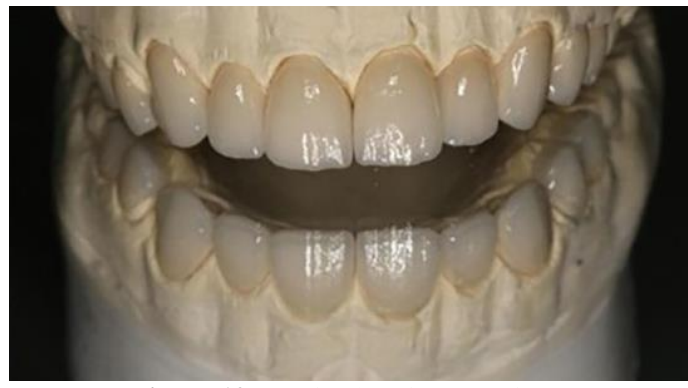

Figura 12: Laminados vitrocerâmicos.

No momento de prova para adaptação dos laminados, foi realizado o teste de cor com o cimento resinoso Allcem Veneer Try-In (FGM ${ }^{\circledR}$, DENTSCARE LTDA, Santa Catarina, Brasil), tendo melhor adaptação cromática a cor translúcida (Figura 13). A cimentação definitiva dos laminados se deu pelo preparo dos mesmos e do substrato, como preconiza o fabricante. $\mathrm{O}$ substrato se encontrava livre de qualquer resíduo e isolado relativamente, então foi condicionado por $30 \mathrm{~s}$, com ácido fosfórico a 37\%, Condac 37 (FGM ${ }^{\circledR}$, DENTSCARE LTDA, Santa Catarina, Brasil), lavado e gentilmente seco (Figura 14).

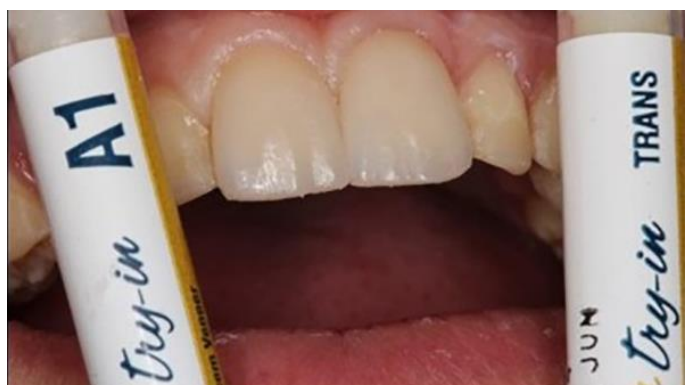

Figura 13: Teste de cor com cimento resinoso Allcem Veneer Try-In.

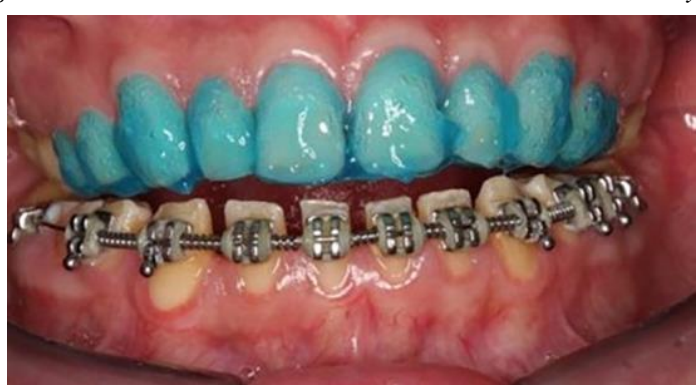

Figura 14: Condicionamento de substrato com ácido fosfórico 37\% por 30segundos.

O processo seguiu com a aplicação de duas camadas de adesivo Ambar (FGM®, DENTSCARE LTDA, Santa Catarina, Brasil), friccionadas por $10 \mathrm{~s}$, individualmente, sem foto ativação (Figura 15). Já os laminados foram preparados condicionando sua 
superfície interna com ácido hidrofluorídrico a 10\%, Condac Porcelana (FGM®, DENTSCARE LTDA, Santa Catarina, Brasil), durante $20 \mathrm{~s}$, lavando, secando, posterior aplicação de silano, Prosil (FGM ${ }^{\circledR}$, DENTSCARE LTDA, Santa Catarina, Brasil) por $15 \mathrm{~s}$ e por fim aplicação de duas camadas de adesivo Ambar, friccionadas por 10 segundos (Figuras 16 e 17).

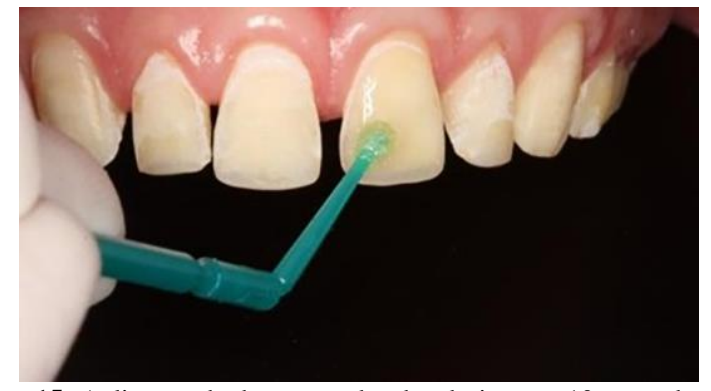

Figura 15: Aplicação de duas camadas de adesivo por 10 segundos cada, sem foto ativação.

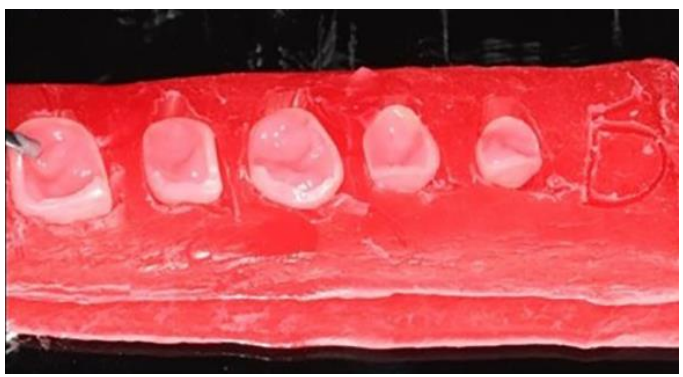

Figura 16: Condicionamento dos laminados com ácido hidro fluorídrico $10 \%$ por 20 segundos.

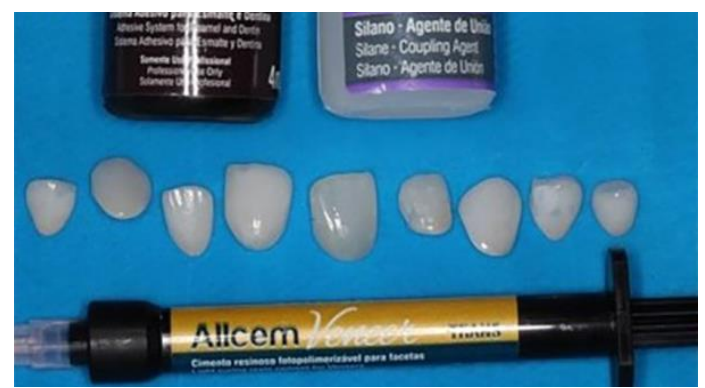

Figura 17: Materiais para preparo e cimentação.

Após o tratamento das superfícies, o cimento resinoso fotopolimerizavel Allcem Veneer (FGM®, DENTSCARE LTDA, Santa Catarina, Brasil) translúcido foi aplicado na face interna dos laminados, e estes posicionados sobre os preparos, removendo-se o excesso de cimento com sonda exploradora e fio dental (Figura 18).

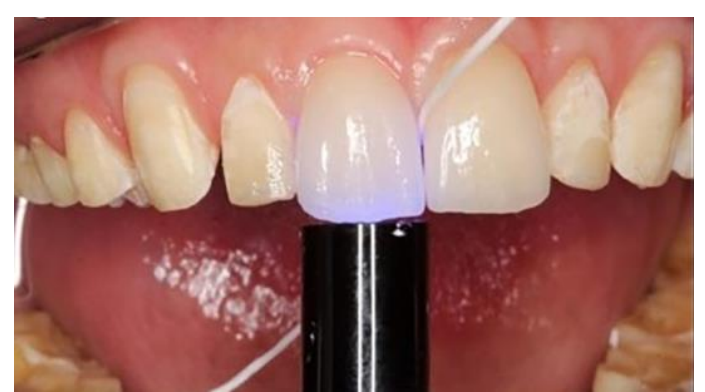

Figura 18: Cimentação das peças dos elementos 11 e 21

As margens da faceta foram protegidas com gel de glicerina e fotoativadas por $40 \mathrm{~s}$, bem com a face vestibular. Os laminados foram cimentados aos pares, inicialmente sobre os dentes 11 e 21, garantindo o alinhamento vertical com o plano sagital, seguindo os 12 e 22 assim por diante (Figura 19). Antes da fase de acabamento e polimento, o teste fonético foi executado e o selamento passivo labial foi observado. Todas as interferências oclusais foram diagnosticadas e ajustadas. No arco inferior os procedimentos se deram da mesma maneira que no arco superior (Figuras 20 a 25).
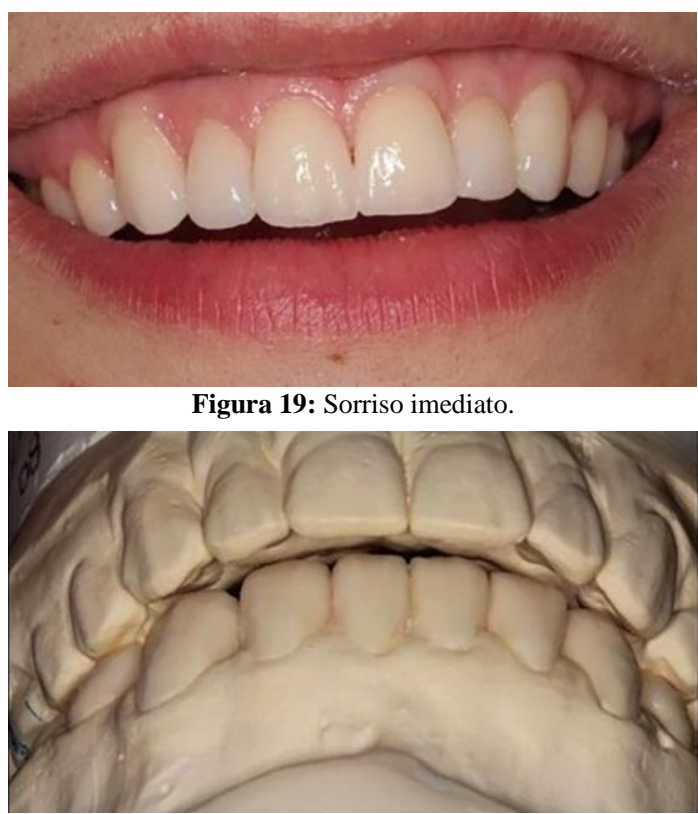

Figura 20: Enceramento diagnóstico inferior.

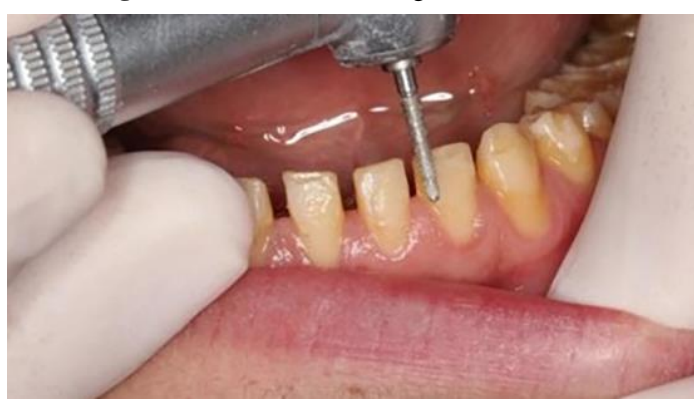

Figura 21: Confecção de bisel.

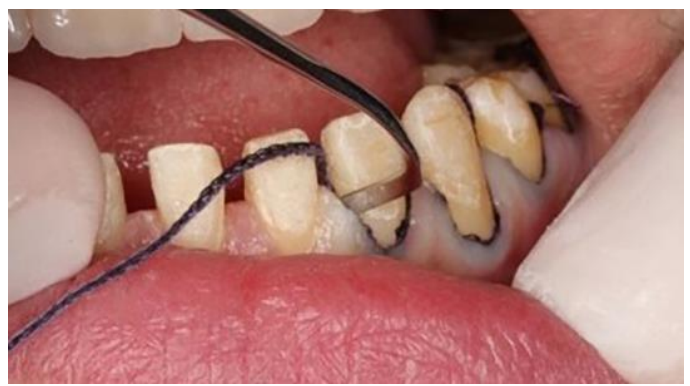

Figura 22: Afastamento gengival.

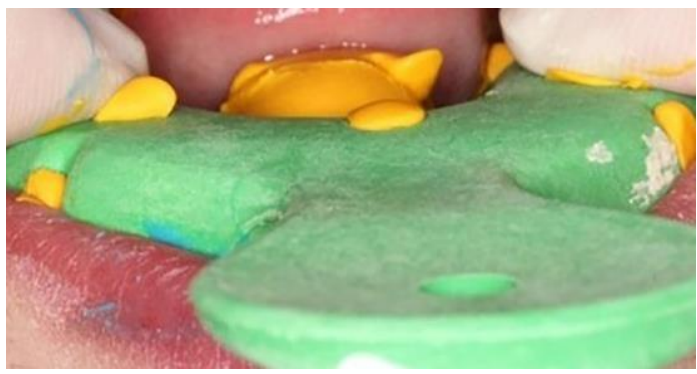

Figura 23: Moldagem com silicone de adição. 


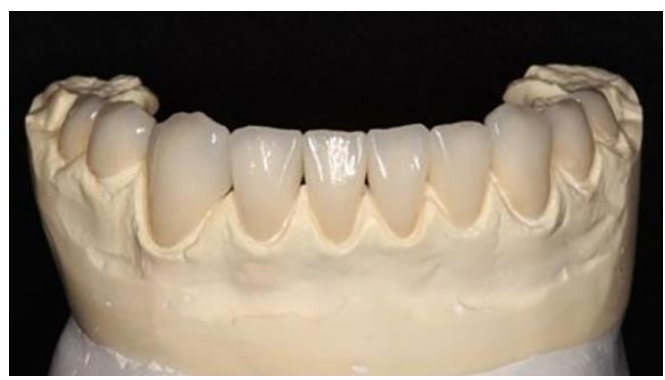

Figura 24: Laminados vitrocerâmicos inferiores.

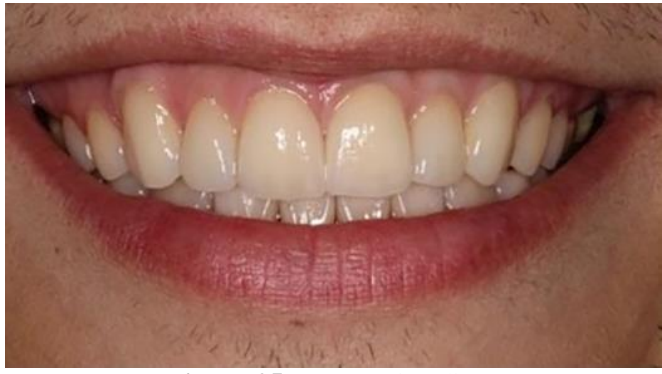

Figura 25: Aspecto imediato.

Como na primeira etapa o aparelho fixo foi removido e o paciente encaminhado para a confecção de aparelho removível, para a manutenção dos espaços. Foi assim confeccionada placa de Hawley para proteção e estabilização do caso. Três consultas de controle foram realizadas, onde foram avaliadas a estabilidade cromática, adaptação da peça e possíveis ajustes. Pode-se observar leve discrepância entre as linhas médias dos incisivos superiores e inferiores (Figura 26).

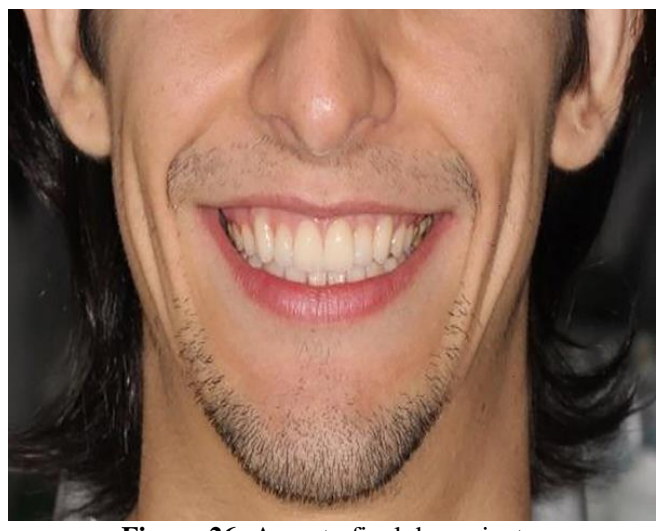

Figura 26: Aspecto final do paciente.

\section{DISCUSSÃO}

Os diastemas se apresentam como um dos desafios mais comuns na odontologia restauradora. ${ }^{11}$ Tal condição é definida como um espaço médio de $0.5 \mathrm{~mm}$, entre as superfícies interproximais dos dentes anteriores ${ }^{12}$. A combinação do correto diagnóstico aliado ao planejamento multidisciplinar consiste na melhor forma no tratamento para casos de múltiplos diastemas ${ }^{13}$. No caso exposto, houve o alinhamento dentário prévio, favorecendo uma abordagem reabilitadora estética e funcional conservadora ${ }^{14}$. Dessa forma, facilitando a harmonia entre dentes, arcos e face, estabelecendo uma oclusão funcional e estável.

A conservação de estruturas dentais sadias é o principal objetivo da odontologia restauradora. $\mathrm{Na}$ odontologia moderna os laminados cerâmicos são as opções mais conservadoras para alcançar as demandas estéticas dos pacientes ${ }^{4}$. O material eleito para o caso foi a vitro-cerâmica reforçada com dissilicato de lítio, devido a suas propriedades ópticas, como o baixo índice de refração, e boa adesividade com o substrato dentário ${ }^{15}$, além de apresentar um conteúdo cristalino maior que outras cerâmicas e uma menor porosidade ${ }^{16}$. Por fim, essa cerâmica é um material biocompatível, garantindo a saúde gengival em longo tempo ${ }^{17}$. Mesmo com um alto conteúdo cristalino, a vitrocerâmica apresenta translucidez suficiente para restaurar bordas incisais e sua estrutura permite resistir às forças de lateralidade e protrusão.

O preparo para os laminados se limita ao esmalte, o que aumenta sua união adesiva. Entretanto, o tipo de cerâmica deve ser conhecido para se estabelecer o correto protocolo de cimentação $^{18}$. No caso do laminado vitrocerâmico, com até $1,5 \mathrm{~mm}$ de espessura, o cimento resinoso fotoativado é a alternativa correta. Materiais com espessura superiores a $1,5 \mathrm{~mm}$ o grau de conversão do cimento pode ser alterado dependendo do tipo de cerâmica e sua opacidade ${ }^{19}$. Cimentos duais não são indicados devido a altas variações cromáticas a longo prazo, fato atribuído a canforoquinonas não ativadas e moléculas de aminas terciárias oxidadas não solubilizadas $^{20}$.

Pela complexidade do caso, o planejamento objetivando a harmonia entre as estruturas do sorriso e a face, baseou-se na proporção áurea ${ }^{10}$. A proporção áurea implica em uma redução progressiva, mésiodistal, de $62 \%$ entre as larguras dos dentes, possibilitando uma construção estética agradável, percebida por vista frontal ${ }^{6}$. A grade de Levin ${ }^{6}$ serve de instrumento para a avaliação da amplitude do sorriso, favorecendo um resultado harmônico e mais específicos $^{6,21}$. Mesmo que a proporção áurea possa ser usada como guia para planejamento, as características individuais de cada paciente devem ser levadas em conta na reconstrução do sorriso. ${ }^{7}$ Uma vez que o clínico conheça e saiba aplicar regras estéticas, levando em conta o equilíbrio entre macro e micro estética, os resultados cosméticos se aproximam do natural ${ }^{22}$.

Apesar da leve discrepância entre a linha média dos incisivos centrais superiores e inferiores, a percepção de estética e harmonia não é quebrada. Discrepâncias de até $2 \mathrm{~mm}$ entre as linhas médias são aceitáveis ${ }^{23}$. Para olhos leigos diferenças até $4 \mathrm{~mm}$ podem não ser percebidas no dia a dia ${ }^{24^{3}}$.

\section{CONSIDERAÇÕES FINAIS}

Um sorriso natural e harmônico vai depender de planejamento integrado, diagnóstico corretos e conhecimento dos materiais e técnicas empregadas. 
Para casos complexos, a proporção áurea, quando alinhada com os conceitos de macro e micro estética, pode levar ao sucesso estético.

A associação de ortodontia prévia a instalação dos laminados permite uma melhor harmonia estética e oclusal, garantindo uma longevidade do tratamento. Opções restauradoras como laminados vitrocerâmicos reforçados com dissilicato de lítio permitem reabilitações extensas minimamente invasivas.

\section{REFERÊNCIAS}

1. Soares GP, Silva FAP, Lima DANL, Paulillo LAMS, Lovadino JR. Prevalência da proporção áurea em indivíduos adultos-jovens. Rev odonto ciênc. 2006;21:346-50.

2. Higashi C, Amaral RC, Hilgenberg SP, Gomes JC, Hirata R, Loguercio R, et al. Finalização estética em dentes anteriores pós tratamento ortodôntico: relato de caso clínico. Int $\mathrm{J}$ Bras Dent. 2007;3:388-98.

3. Kalia A, Mirdehghan N, Khandekar S, Patil W. Multi-disciplinary approach for enhancing orthodontic esthetics - case report. Clin Cosmet Investig Dent. 2015;13:83-9.

4. Otani T, Raigrodski AJ, Mancl L, Kanuma I, Rosen J. In vitro evaluation of accuracy and precision of automated robotic tooth preparation system for porcelain laminate veneers. J Prosthet Dent. 2015;114:229-35.

5. BaratierI LN. Estética: restaurações adesivas diretas em dentes anteriores fraturados. São Paulo: Santos Editora; 1998.

6. Levin, EI. Dental esthetics and the golden proportion. J Prosthet Dent. 1978;3:244-52.

7. Siqueira PM, Nahsan FPS, Naufel FS, Formighieri LA, Schmitt VL. Incidência da proporção áurea regressiva após tratamento ortodôntico. Rev Odontol Bras Central. 2012;21:515-18.

8. Melo GFB, Menezes Filho PFM. Proporção áurea e sua relevância para a odontologia estética. Int $\mathbf{J}$ Dent. 2008;7:234-238.

9. Oliveira VLR. Estudo da proporção áurea entre incisivos centrais. SOTAU R. Virtual Odontol. 2008;5:2-6.

10.Proffit W, Fields HW, Sarver DM. Contemporary orthodontics Fourth edition. Oxford: Elsevier Health Sciences; 2006.

11.Moon JE. Esthetic restorations of maxillary anterior teeth with orthodontic treatment and porcelain laminate veneers: a case report. J Adv Prosthodont. 2010;2:61-63.

12. Keene HJ. Distribution of diastemas in the dentition of man. Am J Phys Anthropol. 1963;21:437-41.

13.Nagalakshmi S, Sathish R, Priya K, Dhayanithi D. Changes in quality of life during orthodontic correction of midline diastema. J Pharm Bioallied Sci. 2014;6:162-64.

14.Jacobson N, Frank CA. The myth of instant orthodontics: an ethical quandary. J Am Dent Assoc. 2008;139:224-34.

15.Bona AD. Bonding to ceramics: scientific evidences for clinical dentistry. São Paulo: Artes Médicas; 2009. p. 91-132.

16.Griggs JA. Recent advances in materials for allceramic restorations. Dent Clin North Am. 2007;51:713-27.

17.Gurel G, Sesma N, Calamita MA, Coachman C, Morimoto S. Influence of enamel preservation on failure rates of porcelain laminate veneers. Int $\mathbf{J}$ Periodontics Restorative Dent 2013;33:31-9.

18. Vargas MA, Bergeron C, Diaz-Arnold A. Cementing all-ceramic restorations: recommendations for success. J Am Dent Assoc. 2011;142:20-24.

19.Runnacles P, Correr GM, Baratto Filho F, Gonzaga CC, Furuse AY. Degree of conversion of a resin cement light-cured through ceramic veneers of different thicknesses and types. Braz Dent J. 2014; 25:38-42.

20. Almeida JR, Schmitt GU, Kaizer MR, Boscato N, Moraes RR. Resin-based luting agents and color stability of bonded ceramic veneers. J Prosthet Dent. 2015;114:272-77.

21.Marubayashi AMW, Shinike, AY, Terada, HH, Kurihara, E, Terada RSS. Avaliação da proporção áurea em pacientes submetidos ou não a tratamento ortodôntico. Rev Dental Press Estét. 2010;7:72-80.

22.Morley J1, Eubank J. Macroesthetic elements of smile design. J Am Dent Assoc. 2001;132:39-45.

23.Johnston CD, Burden DJ, Stevenson MR. The influence of dental to facial midline discrepancies on dental attractiveness ratings. Eur J Orthod. 1999;21:517-22.

24.Kokich VO Jr, Kiyak HA, Shapiro PA. Comparing the perception of dentists and lay people to altered dental esthetics. J Esthet Dent. 1999;11:311-24.

\section{CONFLITO DE INTERESSES}

Os autores declaram não haver conflitos de interesse.

\section{AUTOR PARA CORRESPONDENCIA}

Gabriel Marcondes Castanheira

gmcastanheira@gmail.com

Submetido em 26/11/2018

Aceito em 12/03/2019 